\title{
WORK FUNCTION OF SIMPLE METALS: RELATION BETWEEN THEORY AND EXPERIMENT*
}

\author{
K.F. WOJCIECHOWSKI \\ Institute of Experimental Physics, University of Wroclaw \\ Cybulskiego 36, 50-205 Wrocław, Poland \\ AND H. Bogdanów \\ Institute of Informatics, University of Wroclaw \\ Przesmyckiego 20, 50-151 Wrocław, Poland
}

(Received February 22, 1994; in final form March 22, 1994)

\begin{abstract}
A variational calculation of the face-dependent work function with pseudopotential corrections employed has been performed. Critical analysis of the comparison between the calculated work function values and the experimental polycrystalline data is also given. The polycrystalline work function data may be treated as the mean low-index work function values and compared with the ones calculated. The use of the simple variational method and the Ashcroft pseudopotential for the description of metallic ions leads to good agreement between theory and experiment, and also enables to explain the increasing or decreașing tendency of work function values in different series of the simple metals.
\end{abstract}

PACS numbers: $73.30 .+y, 73.20 .-r$

\section{Introduction}

In the last two decades there appeared several theoretical studies of surface propertics, starting with the pioneering calculations by Lang and $\operatorname{Kohn}[1,2]$ improved later by Perdew and co-workers [4-8]. Also the ab initio calculations of surface properties were performed [3]. Since the $a b$ initio calculations [3] do not give the possibility of a physical interpretation of different contributions to work function (WF) we decided to focus here our attention on the jellium $[1,2]$ and pseudopotential-model treatments [4-8] of this quantity. The main objective of the present study is a critical comparison of polycrystalline and face-dependent

*This work was supported by Wrocław University, grant 2016/W/IFD/93. 
experimental work function data of simple metals with the calculated values, and the analysis of the observed discrepancies.

We present also a variational calculation of WF which complements the previous ones. We take into account the difference of the potential $\delta v(r)$ between the pseudopotential of a lattice of ions and the electrostatic potential of the jellium positive background.

The work function is a most fundamental electronic property of a metallic surface and its determination is of great importance in the understanding of a wide range of surface phenomena. A series of review papers have been published, devoted both to experimental and theoretical aspects of WF. In 1949 an excellent article was published by Herring and Nichols [9], which reviewed the thermionic emission method of WF measurements. More recent techniques of WF measurement are briefly described by Agura and Murata [10]. The general aspects of experimental and theoretical problems connected with WF are given in several reviews [11-13]. Fomenko and Podchernayeva [14] tabulated measured WF data for many elements and compounds, and recommended selected values. The compilation of selected WF data, mainly derived from measurements on polycrystalline samples, were listed by Michaelson [15].

In the case of some metals the polycrystalline WF data given by Michaelson [15] differ considerably from those recommended by Fomenko and Podchernayeva [14], and as a rule the former ones are greater than the latter. In this situation we decide to give (in Tables II and III) both WF data recommended by those authors.

The paper is organized as follows. Section 2 is devoted to a brief review of the work function theory, based on the jellium model and on the Ashcroft pseudopotential and gives original separation of pure jellium part of the work function. In Sec. 3 we discuss the so-called polycrystalline or total WF measured experimentally and its relation to the quantity determined theoretically which may be compared with the former one. A discussion of the existing face-dependent WF data of simple metals is also given in this section. Section 4 presents results of our variational calculations employing the averaged pseudopotential contribution to WF. Section 5 contains a summary and discussion of the collected results. Our conclusions are given in Sec. 6 .

\section{Brief outline of work function theory}

\subsection{Jellium parl of work function}

Treating the semi-infinite metal as the ions smeared into a positive background (jellium) the work function may be expressed as follows $[2,3]$ :

$$
\varphi_{\mathrm{u}}=4 \pi \int_{-\infty}^{\infty} x[n(x)-\bar{n} \theta(-x)] \mathrm{d} x-\left[\varepsilon_{\mathrm{F}}(\bar{n})+\mu_{\mathrm{xc}}(\bar{n})\right],
$$

where

$$
\begin{aligned}
& \varepsilon_{\mathrm{F}}(\bar{n})=k_{\mathrm{F}}^{2} / 2+\mu_{\mathrm{xc}}(\bar{n}), \\
& \mu_{\mathrm{xc}}(\bar{n})=\frac{\partial}{\partial \bar{n}}\left[\bar{n} \varepsilon_{\mathrm{xc}}(\bar{n})\right],
\end{aligned}
$$




$$
\varepsilon_{\mathrm{xc}}(\bar{n})=-\frac{3 k_{\mathrm{F}}}{4 \pi}+\varepsilon_{\mathrm{c}}(\bar{n})
$$

$\varepsilon_{\mathrm{c}}(\bar{n})$ is the correlation energy and [33] $\bar{n}=k_{\mathrm{F}}^{3} / 3 \pi^{2}=3 / 4 \pi r_{\mathrm{s}}^{3}$.

The expression (2.1) depends only on the mean electron density $\bar{n}$. The density functional theory, first applied to surface by Lang and Kohn [2, 3], allows to find self-consistent potentials and electron density profile $n(x)$ at the surface for simple metals within the jellium model and local density approximation (LDA). Having $n(x)$ one can calculate from Eq. (2.1) the jellium part of work function $\varphi_{\mathrm{u}}$.

The self-consistent calculations of $\varphi_{\mathbf{u}}$ done by Lang and Kohn as a function of the density parameters $r_{\mathrm{s}}$ are presented in Fig. 1 by dotted line. These

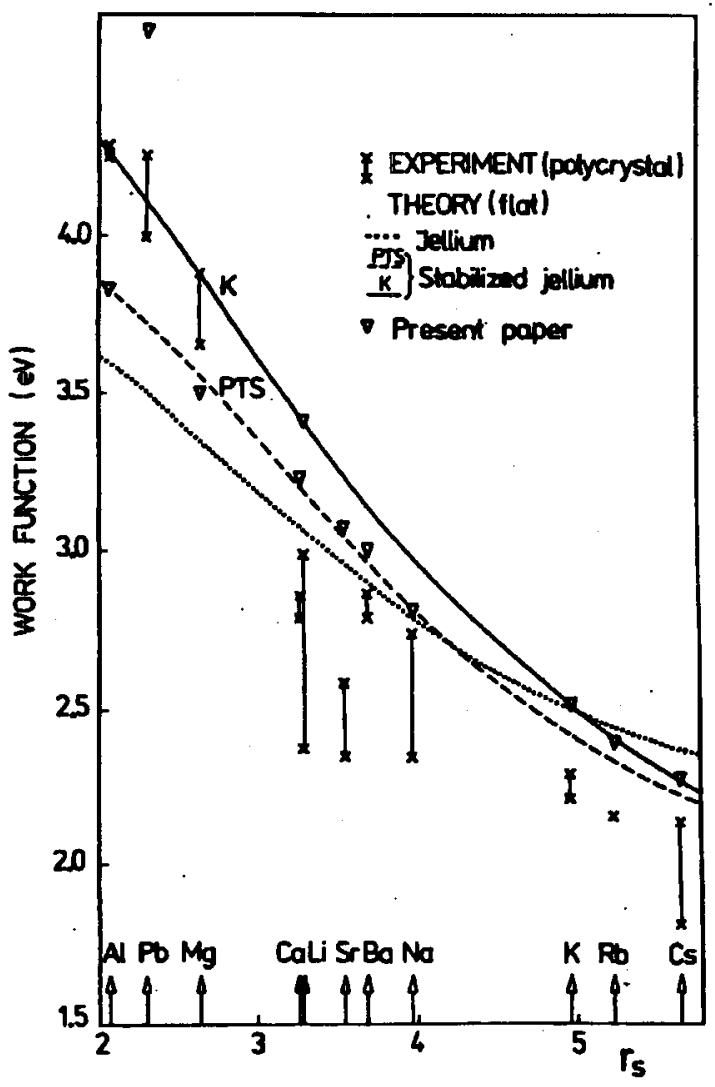

Fig. 1. Comparison of the flat work function values (continuous lines) and present results (triangles) with the experimental polycrystalline data (crosses).

calculations are based, however, on LDA and give the exponential decay of the exchange-correlation potential outside the jellium surface, instead of the correct asymptotics of the classical image-like potential [16].

Comparing experimental values of work function with $\varphi_{u}$ one can conclude that the theoretical values of the jellium part of work function are in agreement 
with the experimental values only qualitatively and some corrections to the simple jellium calculations are needed in order to obtain a better agreement.

\subsection{Work function of the "real" metal bounded by the flat surface}

The jellium model is unstable, it gives negative surface energy (for $r_{s} \leq 2$ ) and bulk modulus (for $r_{\mathrm{s}} \geq 5$ ). To rectify these deficiencies Lang and Kohn [2] reintroduced $\delta v(r)$, the difference between the pseudopotentials of the semi-infinite lattice of ions and the electrostatic potential of the semi-infinite uniform positive background, as a first-order perturbation. The average values of this difference $\langle\delta v(\boldsymbol{r})\rangle_{\text {av }}$ between pseudopotentials of the semi-infinite uniform positive background are often a large fraction of the free-electron Fermi energy and cannot be treated perturbatively. Monnier and Perdew [4] have proposed, therefore to calculate the difference potential $\delta v(r)$ variationally.

In order to introduce the average pseudopotential contribution let us write the expression for the work function in the form

$$
\varphi_{\mathrm{fl}}=\varphi_{\mathrm{u}}(\bar{n})+\varphi_{\mathrm{ps}}^{\mathrm{b}}\left(\bar{n}, r_{\mathrm{c}}\right)
$$

where $\varphi_{\mathrm{u}}$ is the jellium contribution*

$$
\bar{\phi}_{\mathrm{u}}=4 \pi \int_{-\infty}^{\infty} x\left[n_{\mathrm{f}}(x)-n_{+}(x)\right] d x-\left[\varepsilon_{\mathrm{F}}(\bar{n})+\mu_{\mathrm{xc}}(\bar{n})\right]
$$

and

$$
\varphi_{\mathrm{ps}}^{\mathrm{b}}\left(\bar{n}, r_{\mathrm{c}}\right)=-\langle\delta v\rangle_{\mathrm{av}}
$$

is the average (bulk) pseudopotential contribution, $r_{\mathrm{c}}$ being the core radius in Ashcroft's pseudopotential.

In Eq. (2.3), $n_{\mathrm{f}}(x)$ denotes the electron density profile of semi-infinite jellium. In other words, $n_{\mathrm{f}}(x)$ denotes the electron density profile of semi-infinite jellium bounded by the uncorrugate flat surface.

The bulk contribution $\langle\delta v\rangle_{\text {av }}$ to the average of $\delta v(r)$ over the volume of the semi-infinite crystal may be calculated $[4,8]$ assuming that the Wigner-Seitz polyhedrals can be replaced by the spheres of the radius $r_{0}=z^{1 / 3} r_{\mathrm{s}}$ and accepting the Ashcroft $[17,18]$ empty-core pseudopotential $w(r)$ for the interaction between an ion of charge $z$ :

$$
w(r)=\left\{\begin{array}{cc}
-\frac{z}{r}, & r>r_{\mathrm{c}}, \\
0, & r<r_{\mathrm{c}} .
\end{array}\right.
$$
tial is

The average value of repulsive or non-Coulonbic part of the pseudopoten-

$$
\bar{w}_{\mathrm{R}}=\frac{1}{z} \int_{0}^{r_{\mathrm{c}}} \mathrm{d} r w(r)=\frac{1}{z} \int_{0}^{r_{\mathrm{c}}} \mathrm{d} r 4 \pi r^{2} \bar{n} \frac{z}{r}=2 \pi \bar{n} r_{\mathrm{c}}^{2}
$$

and the average of $\delta v(\boldsymbol{r})$ over the volume of the semi-infinite crystal is

$$
\langle\delta v\rangle_{\mathrm{av}}=\frac{1}{d} \int_{-2 d}^{-d} \mathrm{~d} x \delta v(x)=\bar{w}_{\mathrm{r}}-\frac{\pi e^{2} \bar{n}}{6} d^{2},
$$

where $d$ is the distance between crystal planes.

*Atomic units (a.u.) are used unless otherwise stated. 
From Eqs. (2.5) and (2.6) it is seen that $\langle\delta v\rangle_{\text {av }}$ contains both bulk and surface contributions, which can be separated as follows. Let us divide the bulk metal into neutral Wigner-Seitz cells drawn around each ion. For one such a cell let $\delta v_{w s}(r)$ be the sum of the pseudopotential from the ion in this cell and the electrostatic potential of the uniform electronic density contained within it. With the convention that $\delta v_{w s}(r)$ from this cell vanishes far outside it, we find [4]

$$
\delta v_{\mathrm{ws}}(r) \approx\left(-\frac{z e^{2}}{r}+w_{\mathrm{R}}(r)+\frac{3}{2} \frac{z e^{2}}{r_{0}}-\frac{z e^{2} r^{2}}{2 r_{0}^{3}}\right) \theta\left(r_{0}-r\right),
$$

and

$$
\left\langle\delta v_{\mathrm{ws}}\right\rangle_{\mathrm{av}} \approx \bar{w}_{\mathrm{R}}-\frac{3 z e^{2}}{10 r_{0}}=2 \pi \bar{n} r_{\mathrm{c}}^{2}-\frac{3 z e^{2}}{10 r_{0}}
$$

As we see the $\left\langle\delta v_{\mathrm{ws}}\right\rangle_{\mathrm{av}}$ is the bulk contribution to $\langle\delta v\rangle_{\mathrm{av}}$.

Under these assumptions the sum of repulsive part of pseudopotential, the average (electrostatic) energy of collection of point ions embedded in a uniform negative background of density $\bar{n}$, and the electrostatic self-energy of this background in the sphere of radius $r_{0}$ are given by Eq. (2.8).

In conclusion one can say that subtracting from the jellium energy functional the electrostatic self-repulsion energy of positive background and adding the interaction between the electrons and potential $\delta v(r)$ transforms approximately the jellium to the real metal [8].

In this approximation the real-metal model total energy, as a functional of electron density is

$$
\begin{gathered}
E_{\mathrm{ps}}\left[n, n_{+}\right]=E_{\mathrm{jell}}\left[n, n_{+}\right]+\left(\varepsilon_{\mathrm{M}}+\bar{w}_{\mathbf{R}}\right) \int \mathrm{d} \boldsymbol{r} n_{+}(r) \\
+\left\langle\delta v_{\mathrm{ws}}\right\rangle_{\mathrm{av}} \int \mathrm{d} \boldsymbol{r} \theta(\boldsymbol{r})\left[n(r)-n_{+}(r)\right],
\end{gathered}
$$

where $E_{\text {jell }}$ is the standard energy functional for the jellium, $\varepsilon_{\mathrm{M}}=-9 z / 10 r_{0}$ is the Madelung energy, $\bar{w}_{\mathrm{R}}$ is given by Eq. (2.5) and $\theta(\boldsymbol{r})=1$ inside the metal and 0 outside.

We see therefore that the transformation of the jellium-model to the pseudopotential-model, which simulates a real metal, leads to the dependence of the face-independent part of work function on the individual metal specificity represented in the above picture of metal by the core radius $r_{c}$ of the Ashcroft pseudopotential, usually fitted to some measured properties.

It is to be noted that now we have used average $\delta v(r)$ value over the Wigner-Seitz cell and confined the semi-infinite metal to the region $x \leq 0$, therefore work function is given by the following expression [8]:

$$
\varphi_{\text {flat }}=\varphi_{\mathrm{u}}+\left\langle\delta v_{\mathrm{ws}}\right\rangle_{\mathrm{av}} \int_{-\infty}^{0} \mathrm{~d} x \frac{1}{n} \frac{\mathrm{d} n(x)}{\mathrm{d} x} .
$$




\subsection{Face-dependent part of work function}

The work function of a real metal is strongly face-dependent and may be written in the form

$$
\varphi_{i}=\varphi_{\mathrm{f}}+\delta \varphi_{i},
$$

where $\varphi_{\mathrm{f}}$ is the face-independent and $\delta \varphi_{i}$ face-dependent part of work function.

The face-dependent part $\delta \varphi_{i}$ in the case of the semi-infinite metal may be written in the form

$$
\delta \varphi_{i}=4 \pi \int_{-\infty}^{\infty} x\left[n_{i}(x)-n_{\mathrm{f}}(x)\right] \mathrm{d} x+D_{\mathrm{cl}}^{i},
$$

where $D_{\mathrm{cl}}^{i}$ is the contribution to the surface dipole barrier that arises from the distortion of Wigner-Seitz cells which occurs in the classical cleavage under stress, and $n_{i}(x)$ is the electron charge distribution which arises from the subsequent relaxation of the electron density [4].

Since the charge neutrality condition, both for the semi-infinite jellium and for the semi-infinite real metal, has to be satisfied and because the main contribution to the value of the integral in (2.13) comes from the surface region where the Friedel oscillations are great, one can state that this integral is small and approximately write

$$
\delta \varphi_{i}=D_{\mathrm{cl}}^{i}
$$

Denoting the surface contribution to $\langle\delta v\rangle_{\mathrm{av}}$ by $-D_{\mathrm{cl}}^{i}$, we can write

$$
\langle\delta v\rangle_{\mathrm{av}}=\left\langle\delta v_{\mathrm{ws}}\right\rangle_{\mathrm{av}}-D_{\mathrm{cl}}^{i},
$$

and employing Eqs. (2.7) and (2.8) one obtains

$$
D_{\mathrm{cl}}^{i}=-\frac{3 z}{10 r_{0}}\left[1-\frac{5}{12}\left(\frac{d}{r_{0}}\right)^{2}\right] \equiv-\frac{z^{2 / 3}}{8 r_{\mathrm{s}}} F\left(\mathrm{~d}_{i}^{2} / r_{0}^{2}\right),
$$

where $F(x)=\frac{12}{5}-z$.

\section{TABLE I}

Values of the factor $F$ in Eq. $(2.16)^{a}$.

\begin{tabular}{c|c|c|c}
\hline \hline fcc & $F$ & bcc & $F$ \\
\hline$(111)$ & 0.2173 & $(110)$ & 0.3376 \\
$(100)$ & 0.7631 & $(100)$ & 1.3687 \\
$(110)$ & 1.5815 & $(111)$ & 2.0562 \\
\hline hcp & $F(\mathrm{Zn})$ & & $F(\mathrm{Mg})$ \\
$(0001)$ & -0.1982 & & -0.1685 \\
\hline
\end{tabular}

${ }^{a}$ Values of the ratio $d_{i} / r_{0}$ taken from

Table IV of Ref. [4]. 
From Table I, where the values of the factor $F$ are given, we see that $\operatorname{sgn} F(x)= \begin{cases}+, & \text { for fcc and bcc structures, } \\ -, & \text { for hep structure. }\end{cases}$

Therefore we arrive at the final conclusion that

$\varphi_{i}<\varphi_{\mathrm{l}}, \quad$ for $\mathrm{fcc}$ and bec structures,

and

$\varphi_{0001}>\varphi_{\mathrm{A}}, \quad$ for $i=0001$ of hep structure.

\section{Polycrystalline and face-dependent work function}

In experiment we have three "kinds" of work function: (i) the mean or polycrystalline work function $\varphi_{\mathrm{P}}$ obtained from measurement of polycrystalline samples, (ii) the so-called total work function $\varphi_{\mathrm{T}}$, determined by measurements of the total thermoemission current or the field emission current from a single-crystal tip, and (iii) the $\varphi_{h k l} \equiv \varphi_{i}$, measured for a particular crystal $i$-th face with Miller indices $(h k l) \equiv i$.

The polycrystalline surface may be treated as a composition of patches $[6,9,19]$ each patch being a certain cleavage plane of crystals and the $\varphi_{\mathbf{P}}$ may be interpreted as an average of the work function of all exposed patches

$$
\varphi_{\mathrm{P}}=\sum_{i} f_{i} \varphi_{i} / \sum_{i} f_{i}
$$

where $\varphi_{i}$ is the work function of cleavage corresponding to the $i$-th patch, and $f_{i}$ is its weight.

Taking simply $f_{i}=A_{i}, A_{i}$ being the surface of the $i$-th facet, we have [19]

$$
\varphi_{\mathrm{P}}=\sum_{i} A_{i} \varphi_{i} / \sum_{i} A_{i}
$$

More accurate definition of weights $f$ was given by Sahni et al. [6].

When the surface of a sample of given metal is clean enough, and the experiment is performed in the ultrahigh vacuum, measurements of $\varphi_{\mathrm{P}}$ and $\varphi_{\mathbf{T}}$, within the limits of the experimental accuracy, give the same value [13]. Therefore, practically $\varphi_{\mathrm{P}}=\varphi_{\mathrm{T}}$.

However, the comparison of $\varphi_{P}$ with work function calculated theoretically is not simple at least from the point of view of the thermodynamics of a crystal growth, according to which the equilibrium shape of a crystal is governed by the Wulf theorem. On the polycrystalline surface mainly taken as a fraction (of the total considered), the low-index crystal faces are exposed, which have a greater surface energy than the high-index crystal faces. Therefore, for the comparison of the theoretical calculations with the experimental data, it seems appropriate to compare $\varphi_{\mathrm{P}}$ with the mean value of the work functions calculated theoretically for the three lowest-index crystal faces. 
TABLE II Calculated face-dependent and polycrystalline work functions (eV) of monovalent simple metals.

\begin{tabular}{|c|c|c|c|c|c|c|c|c|c|}
\hline \multirow{3}{*}{ Metal } & \multicolumn{4}{|c|}{ Stabilized jellium } & \multirow{2}{*}{\multicolumn{2}{|c|}{$\begin{array}{c}\text { Present } \\
\text { paper }\end{array}$}} & \multirow{2}{*}{\multicolumn{3}{|c|}{ Experiment }} \\
\hline & \multicolumn{2}{|c|}{ variat. $^{a}$} & \multicolumn{2}{|c|}{ self-cons. $^{b}$} & & & & & \\
\hline & $\varphi_{i}$ & $\left\langle\varphi_{i}\right\rangle$ & $\varphi_{i}$ & $\left(\varphi_{i}\right)$ & $\varphi_{i}$ & $\left\langle\varphi_{i}\right\rangle$ & $\varphi_{i}$ & \multicolumn{2}{|c|}{$\left\langle\varphi_{\mathrm{P}}\right\rangle$} \\
\hline $\mathbf{L i}$ & & & & & $r_{c}=$ & 1.06 & & & \\
\hline flat & 3.18 & 2.97 & 2.36 & 3.08 & 3.44 & 3.10 & & $2.38^{e}$ & $2.9^{f}$ \\
\hline 110 & 3.09 & & 3.27 & & 3.32 & & & & \\
\hline 100 & 2.92 & & 3.03 & & 3.04 & & & & \\
\hline 111 & 2.90 & & 2.93 & & 2.94 & & & & \\
\hline $\mathbf{N a}$ & & & & & $r_{\mathrm{c}}=$ & 1.758 & & & \\
\hline flat & 2.83 & 2.60 & 2.94 & 2.71 & 2.84 & 2.62 & & $2.35^{e}$ & $2.75^{f}$ \\
\hline 110 & 2.75 & & 2.88 & & 2.76 & & $2.90^{c}$ & & \\
\hline 100 & 2.58 & & 2.66 & & 2.60 & & & & \\
\hline 111 & 2.54 & & 2.59 & & 2.57 & & & & \\
\hline $\mathbf{K}$ & & & & & $r_{c}=$ & 2.23 & & & \\
\hline flat & 2.45 & 2.25 & 2.50 & 2.30 & 2.56 & 2.30 & & $2.22^{e}$ & $2.30^{f}$ \\
\hline 110 & 2.37 & & 2.44 & & 2.43 & & $2.45^{d}$ & & \\
\hline 100 & 2.21 & & 2.27 & & 2.26 & & 2.06 & & \\
\hline 111 & 2.17 & & 2.19 & & 2.21 & & 1.85 & & \\
\hline $\mathbf{R b}$ & & & & & $r_{c}=$ & 2.40 & & & \\
\hline flat & 2.36 & 2.16 & 2.39 & 2.21 & 2.41 & 2.19 & & $2.16^{e f}$ & \\
\hline 110 & 2.28 & & 2.33 & & 2.33 & & & & \\
\hline 100 & 2.12 & & 2.17 & & 2.15 & & & & \\
\hline 111 & 2.00 & & 2.12 & & 2.09 & & & & \\
\hline Cs & & & & & $r_{c}=$ & 2.40 & & & \\
\hline flat & 2.24 & 2.05 & 2.26 & 2.08 & 2.28 & 2.07 & & $1.81^{e}$ & $2.14^{f}$ \\
\hline 110 & 2.17 & & 2.19 & & 2.21 & & & & \\
\hline 100 & 2.01 & & 2.04 & & 2.03 & & & & \\
\hline 111 & 1.97 & & 2.01 & & 1.98 & & & & \\
\hline
\end{tabular}

${ }^{a}$ Perdew et al. [8], ${ }^{b}$ Kiejna [28], ${ }^{c}$ Anderson et al. [34], ${ }^{d}$ Błaszczyszyn et al. [26], ${ }^{e}$ Fomenko and Podchernayeva [14], ${ }^{f}$ Michaelson [15]. 
From the said above it follows that face-dependent WF may be represented by a sum

$$
\varphi_{i}=\varphi_{\mathrm{f}}+\delta \varphi_{i}
$$

where $\varphi_{\mathrm{A}}$ is the face-independent part of work function and $\delta \varphi_{i}$ is face-dependent part of work function.

According to Eqs. (9.20) and (9.21) the mean work function of a polycrystalline surface is

$$
\left\langle\varphi_{i}\right\rangle_{i}=\varphi_{\mathrm{fH}}+\sum_{i} A_{i} \delta \varphi_{i} / \sum_{i} A_{i}
$$

In the case of metals one can suppose that the (111), (100) and (110) faces all have essentially the same surface energy [20] and therefore $A_{111} \approx A_{100} \approx A_{110}$ which, in connection with the said above, leads to the following approximate expression for average WF:

$$
\left\langle\varphi_{i}\right\rangle \approx \varphi_{\mathrm{f}}+\frac{1}{3}\left(\delta \varphi_{111}+\delta \varphi_{100}+\delta \varphi_{110}\right),
$$

giving the work function value that may be compared with the experimental polycrystalline data $\left(\varphi_{\mathrm{P}}\right)$.

The theoretically calculated, from Eq. (3.3), face-dependent values of work function can be directly compared with the experimental data of $\varphi_{i}$ but the latter, for clean metallic single-crystals, are very poor and uncertain (e.g. in the case of alkali metals). From the midst of simple metals, the face-dependent experimental data of work function only for low-index aluminium crystal-faces are available but they differ between each other in the trend of increasing of their values (see Table III). The trend of Eastman and Mee's [21] values is consistent with the argumentation given by Smoluchowski [22], while the trend of Grepstad et al. [23] values is in complete disagreement with these arguments.

For alkali metals there is lack of measured values of $\varphi_{i}$ from a single-crystal because of great difficulties in preparation of such samples. However, investigations of the epitaxial growth of these metals on tungsten have shown [24] that monolayers of alkali metals formed on a single-crystal of tungsten have the same structure as the corresponding faces of a single-crystal of alkali metal. From the analysis of the experimental data [25] it follows that for sodium and potassium $\varphi_{110}>\varphi_{100}>\varphi_{111}$, which is in agreement with the Smoluchowski rule. For cesium adsorbed on tungsten, this trend is very weak [26] and the average nearest-neighbor distance in the cesium-metal (fcc and bcc) monolayers equals $4.92 \AA$ for the saturation coverage, whereas in the bulk metal this distance amounts to $5.25 \AA$ [10]. From this fact it follows that cesium monolayers form densely packed hexagonal (or quasi-hexagonal) lattices (irrespective of the symmetry of the substrates) which do not appear in the bulk cesium. Therefore the mean work function $(\approx 1.9 \div 2 \mathrm{eV})$ measured for cesium monolayers on metals cannot be referred to the work function value of any low-index crystal face of bulk cesium. 
TABLE III

Face-dependent and polycrystalline work functions (eV) of divalent and polyvalent simple metals.

\begin{tabular}{|c|c|c|c|c|c|c|c|c|c|c|}
\hline \multirow{3}{*}{ Metal } & \multicolumn{4}{|c|}{ Stabilized jellium } & \multirow{2}{*}{\multicolumn{2}{|c|}{$\begin{array}{l}\text { Present } \\
\text { paper }\end{array}$}} & \multirow{2}{*}{\multicolumn{4}{|c|}{ Experiment }} \\
\hline & \multicolumn{2}{|c|}{ variat. $^{a}$} & \multicolumn{2}{|c|}{ self-cons. $^{b}$} & & & & & & \\
\hline & $\overline{\varphi_{i}}$ & $\left\langle\varphi_{i}\right\rangle$ & $\varphi_{i}$ & $\left\langle\varphi_{i}\right\rangle$ & $\varphi_{i}$ & $\left\langle\varphi_{i}\right\rangle$ & \multicolumn{2}{|c|}{$\overline{\varphi_{i}}$} & \multicolumn{2}{|r|}{$\left\langle\varphi_{\mathrm{P}}\right\rangle$} \\
\hline $\mathrm{Al}$ & & & & & $\overline{r_{\mathrm{c}}}=$ & 1.12 & & & & \\
\hline flat & 3.83 & 3.72 & 4.27 & 3.82 & 3.83 & 3.73 & & & $4.25^{e}$ & $4.28^{f}$ \\
\hline 111 & 3.72 & & 4.09 & & 3.73 & & $4.26^{c}$ & $4.24^{d}$ & & \\
\hline 100 & 3.62 & & 3.77 & & 3.63 & & 4.20 & 4.41 & & \\
\hline 110 & 3.81 & & 3.59 & & 3.82 & & 4.06 & 4.28 & & \\
\hline $\mathrm{Pb}$ & & & & & $r_{\mathrm{c}}=$ & 1.12 & & & & \\
\hline flat & 3.73 & 3.62 & 4.09 & 3.64 & 4.64 & 3.87 & & & $4.00^{e}$ & $4.25^{f}$ \\
\hline 111 & 3.59 & & 3.90 & & 4.34 & & & & & \\
\hline 100 & 3.50 & & 3.57 & & 3.76 & & & & & \\
\hline 110 & 3.77 & & 3.46 & & 3.51 & & & & & \\
\hline $\mathrm{Ca}$ & & & & & $r_{\mathrm{c}}=$ & 1.70 & & & & \\
\hline flat & 3.19 & 2.99 & & & 3.24 & 3.01 & & & $2.80^{e}$ & $2.87^{f}$ \\
\hline 111 & 3.10 & & & & 3.14 & & & & & \\
\hline 100 & 2.94 & & & & 2.97 & & & & & \\
\hline 110 & 2.92 & & & & 2.92 & & & & & \\
\hline $\mathbf{S r}$ & & & & & $r_{\mathrm{c}}=$ & 1.682 & & & & \\
\hline flat & 3.03 & 2.83 & & & 3.33 & 2.97 & & & $2.35^{e}$ & $2.59^{f}$ \\
\hline 111 & 2.94 & & & & 3.21 & & & & & \\
\hline 100 & 2.79 & & & & 2.95 & & & & & \\
\hline 110 & 2.75 & & & & 2.76 & & & & & \\
\hline $\mathrm{Ba}$ & & & & & $r_{\mathrm{c}}=$ & 1.984 & & & & \\
\hline flat & 2.96 & 2.74 & & & 3.02 & 2.76 & & & $2.49^{e}$ & $2.7^{f}$ \\
\hline 110 & 2.83 & & & & 2.88 & & & & & \\
\hline 100 & 2.67 & & & & 2.68 & & & & & \\
\hline 111 & 2.73 & & & & 2.72 & & & & & \\
\hline $\mathrm{Zn}$ & & & & & $r_{c}=$ & 1.11 & & & & \\
\hline flat & 3.73 & 3.83 & 4.09 & 4.24 & 3.68 & 3.77 & & & $4.24^{e}$ & $4.33^{f}$ \\
\hline 0001 & 3.83 & & 4.24 & & 3.77 & & $4.9^{f}$ & & & \\
\hline $\mathbf{M g}$ & & & & & $r_{c}=$ & 1.358 & & & & \\
\hline flat & 3.54 & 3.44 & 3.82 & 3.71 & 3.48 & 3.58 & & & & \\
\hline 0001 & 3.44 & & 3.71 & & 3.58 & & $3.8^{g}$ & & $3.64^{e}$ & $3.66^{f}$ \\
\hline
\end{tabular}

${ }^{a}$ Perdew et al. [8], ${ }^{b}$ Kiejna [28], ${ }^{c}$ Eastman and Mee [21], ${ }^{d}$ Grepstad et al. [23], ${ }^{e}$ Fomenko and Podchernayeva [14], ${ }^{f}$ Michaelson [15], ${ }^{g}$ Hayden et al. [35]. 


\section{Jellium with pseudopotential: variational calculation of work function for low-index crystal planes}

Perdew et al. [8] introduce the mentioned in Sec. 2 transformation of jellium to real metal and assume also the determination of the Ashcroft core radius $r_{c}$ of Eq. (2.6) from the bulk stability condition. They name their model: the stabilized jellium. So in stabilized jellium, when $\bar{n}$ is the equilibrium density, the "core radius" $r_{\mathrm{c}}$ is determined unequivocally by this density, i.e. by the density parameter $r_{\mathrm{s}}$ (see eq. (26) of Ref. [8]). This assumption makes that the theory dependent only on one input parameter $r_{\mathrm{s}}$ and the physical quantities involved become continuous functions of this parameter. In the Ashcroft empty-core pseudopotential theory, however, the core radius $r_{\mathrm{c}}$ is fitted to some measured bulk physical properties of individual metal and therefore its particular value characterizes this individual metal.

The experimental polycrystalline $\mathrm{WF}$ is discrete function of the average valence-electron density. Therefore, in order to compare experimental $\varphi_{\mathbf{P}}$ data with theory we do not assume that $r_{c}$ can be determined as a continuous function of $r_{\mathrm{s}}$ such as in the stabilized jellium model [8], but we take the $r_{\mathrm{c}}$ values determined for particular metals as in the pseudopotential theory [18], and employ Eq. (2.16) for $\delta W_{i}$ and Eq. (2.2) for $W_{\mathrm{ff}}$. The core radii $r_{\mathrm{c}}$ are collected in Table IV.

WF may be evaluated from the displaced-profile change-in-self-consistentfield expression [5] in the form [8]

$$
\begin{aligned}
\varphi_{i}= & \phi(\infty)-\phi(0)-\left[t_{\mathrm{s}}(\bar{n})+\varepsilon_{\mathrm{xc}}(\bar{n})\right] \\
& +\left(\left\langle\delta v_{\mathrm{ws}}\right\rangle_{\mathrm{av}}-D_{\mathrm{cl}}^{i}\right) \frac{1}{\bar{n}} \int_{-\infty}^{0} \mathrm{~d} x \frac{\mathrm{d} n(x)}{\mathrm{d} x},
\end{aligned}
$$

where

$$
\phi(x)=\phi(-\infty)+4 \pi \int_{-\infty}^{x} \mathrm{~d} x^{\prime}\left(x^{\prime}-x\right)\left[n\left(x^{\prime}\right)-\bar{n} \theta\left(-x^{\prime}\right)\right]
$$

is the electrostatic potential and $t_{s}(\bar{n})+\varepsilon_{\mathrm{xc}}(\bar{n})$ is the sum of the average kinetic and exchange-correlation energy per valence electron.

For $n(x)$ we employ a simple, Perdew's analytical model [29] in the form [8]

$$
\frac{n(y)}{\bar{n}}= \begin{cases}1+b_{0} \mathrm{e}^{y}+b_{1} \mathrm{e}^{\alpha y}, & y<0, \\ b_{2} \mathrm{e}^{-\beta y}, & y>0,\end{cases}
$$

where $\gamma$ is the variational parameter and

$$
k_{\mathrm{s}}=\left(\frac{4 k_{\mathrm{F}}}{\pi}\right)^{1 / 2}
$$

is the bulk Thomas-Fermi screening wave vector. 


\section{TABLE IV}

The properties of simple metals: the valence $z$, the density parameter $r_{\mathrm{s}}$ and Ashcroft radius $r_{\mathrm{c}}$.

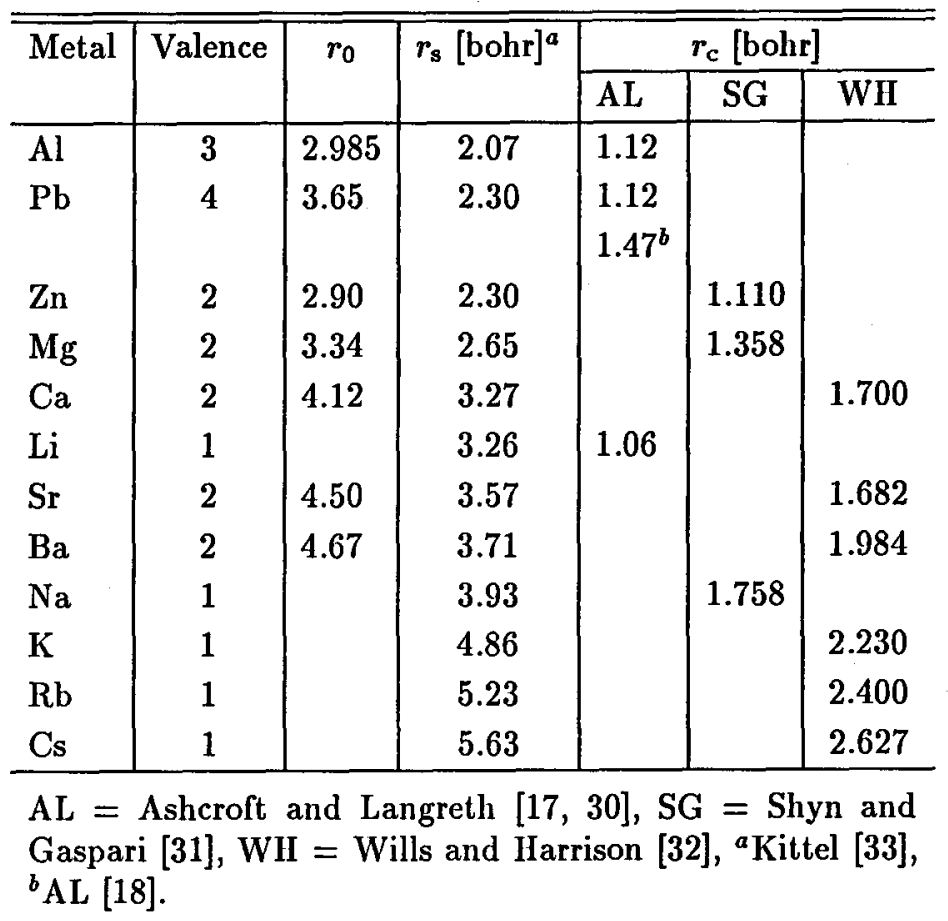

The parameter $\gamma$ is chosen to minimize the surface energy $\sigma$, which for $n(x)$ given by Eq. (3.18) may be written as follows [8]:

$$
\sigma=\frac{k_{\mathrm{F}}^{9 / 2}}{27 \pi^{2}}\left(\frac{a_{\mathrm{es}}}{3 \gamma^{3}}-\frac{a}{\gamma}+\frac{a_{2} \gamma}{k_{\mathrm{F}}}+\frac{a_{4} \gamma^{3}}{3 k_{\mathrm{F}}^{2}}\right)
$$

where

$$
a=a_{0}-\frac{a_{\mathrm{x}}+a_{\mathrm{c}}(\bar{n})}{k_{\mathrm{F}}}+a_{\mathrm{ps}} \frac{\left\langle\delta v_{\mathrm{ws}}\right\rangle_{\mathrm{av}}-D_{\mathrm{cl}}^{i}}{k_{\mathrm{F}}^{2} / 2} .
$$

The values of the constants $a_{\mathrm{cs}}, a_{0}, \ldots a_{\mathrm{ps}}, b_{0}, b_{1}, b_{2}, \alpha$ and $\beta$ appearing in Eqs. (4.3), (4.5) and (4.6), are given in Table III of Ref. [8]. The correlation constant $a_{c}(\bar{n})$ is given by Eq. (44) of Ref. [8].

The variational computations of $n(x)$ and WF were performed both for the low-index crystal planes and for the flat surface. In the latter case we omit in the calculations the term $D_{\mathrm{cl}}^{i}$ that appears in Eqs. (4.6) and (4.1). The corresponding values of parameter $\gamma$ which minimizes $\sigma$ given by Eq. (4.5), are presented in Table V. 
TABLE V

Values of the variational parameter $\gamma$ as determined from the minimum of the surface energy condition for $r_{\mathrm{c}}$ values given in bohrs in parenthesis.

\begin{tabular}{c|c|c|c|c|c|c|c}
\hline \hline Face & $\mathrm{Li}(1.06)$ & $\mathrm{Na}(1.758)$ & $\mathrm{K} \mathrm{(2.23)}$ & $\mathrm{Rb}(2.4)$ & $\mathrm{Cs}(2.627)$ \\
\hline flat & 1.68 & 1.49 & 1.52 & 1.50 & 1.47 \\
110 & 1.62 & 1.42 & 1.42 & 1.42 & 1.44 \\
100 & 1.42 & 1.20 & 1.20 & \multicolumn{2}{|c}{1.21} & \multicolumn{1}{c}{1.02} \\
111 & 1.26 & 1.07 & 1.06 & \multicolumn{2}{|c}{1.06} & 0.59 \\
\hline Face & $\mathrm{Al}$ & $\mathrm{Pb}$ & $\mathrm{Zn}$ & $\mathrm{Mg}$ & $\mathrm{Ca}$ & $\mathrm{Sr}$ & $\mathrm{Ba}$ \\
& $(1.12)$ & $(1.12)$ & $(1.11)$ & $(1.358)$ & $(1.70)$ & $(1.682)$ & $(1.984)$ \\
\hline flat & 1.59 & 1.98 & 1.55 & 1.53 & 1.75 & 1.70 & 1.56 \\
111 & 1.50 & 1.88 & & & 1.50 & 1.63 & 1.50 \\
100 & 1.29 & 1.60 & & & 1.32 & 1.46 & 1.31 \\
110 & 1.06 & 1.21 & & & 1.08 & 1.19 & 1.06 \\
0001 & & & 1.61 & 1.56 & & &
\end{tabular}

\section{Relation between theory and experiment}

The calculated in Sec. 4 values of $\varphi_{i}$ and $\varphi_{f}$ are collected in Tables II and III together with the experimental data of work function measurements for simple metals. The related $\left\langle\varphi_{i}\right\rangle$ values, calculated from Eq. (2.5), are shown in Fig. 2, together with experimental data. Figure 1 represents a comparison between flat-surface calculations and the experimental data.

In Figure 1 the jellium part of work function calculated variationally [8] is represented by dotted line. Dashed and full lines represent the variational [8] and self-consistent [27] calculations for the flat surface of the stabilized jellium respectively. From this figure we observe that calculations done in the framework of stabilized jellium give lower values of work function for larger $r_{s}$ and greater ones for the density parameter $r_{\mathbf{s}}$ less than its jellium values. Generally, this result is in agreement with experimental observation, however for $\mathrm{Li}, \mathrm{Na}$ and for divalent metals: $\mathrm{Ca}, \mathrm{Sr}$, and $\mathrm{Ba}$, the jellium model gives a better agreement with experimental data than the calculations for flat surface performed in the framework of the stabilized jellium model. This disagreement appears probably from the fact that in the stabilized jellium model the core radius $r_{\mathrm{c}}$ is determined from the bulk stability condition and becomes a continuous function of the density parameter $r_{\mathrm{s}}$. As it was mentioned in Sec. 4 the Ashcroft empty-core radius $r_{\mathrm{c}}$ in the pseudopotential theory is fitted to some measured properties of individual metal and therefore its particular value characterizes this individual metal.

In order to check what results from the flat metal model described in Sec. 2.2 we calculated in the previous section the flat work function values rejecting of the assumption of stabilized jellium model that $r_{\mathrm{c}}=f\left(r_{\mathrm{s}}\right)$. Such calculations 


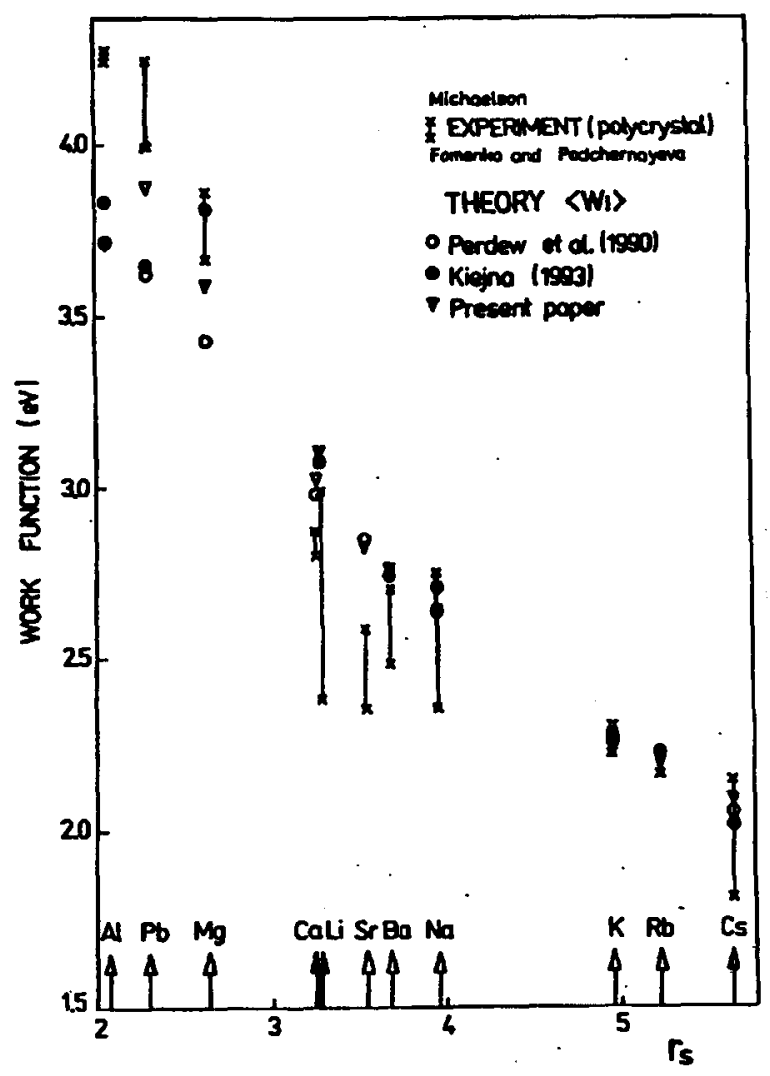

Fig. 2. Comparison of values of the calculated work function and experimental polycrystalline data (crosses).

give results presented in Fig. 1 by triangles. The latter results show that the introduction of the average pseudopotential contribution to the work function calculations gives the best agreement with experiment from among the considered models. The last model for $\mathrm{K}, \mathrm{Rb}$ and $\mathrm{Cs}$ gives a lower work function than the one calculated in the framework of jellium model and greater one for $\mathrm{Al}, \mathrm{Pb}$ and $\mathrm{Mg}$. Moreover, it gives a consistent trend of the observed work functions of $\mathrm{Al}$, $\mathrm{Pb}$ and $\mathrm{Mg}$. The trend of the calculated work functions of $\mathrm{Ca}, \mathrm{Li}, \mathrm{Sr}, \mathrm{Ba}$ and $\mathrm{Na}$ is also better than that resulting from the jellium model and especially from the stabilized jellium model.

In Fig. 2 the mean work function values calculated are compared with the polycrystalline data. It may be seen from this figure that our calculations give quite a good agreement with those data. In the case of $\mathrm{Na}, \mathrm{K}, \mathrm{Rb}$ and $\mathrm{Cs}$ agreement is even better than for the self-consistent calculations done in the framework of the stabilized jellium model. This result shows that the mean surface contribution to work function generally lowers the jellium work function for fcc and bcc metals and raises it for hcp metals. 


\section{Conclusions}

A comparison between the calculated mean values of the work function of the three low-index faces (100), (110) and (111) and the measured polyvalent work function values shows quite a good agreement between theory and experiment. The present calculations prove that the agreement can be even better in some cases if one gives up the assumption of the stabilized jellium that the core radius $r_{\mathrm{c}}$ may be determined from the bulk stability condition. On the basis of the calculations performed and analysis of the results obtained it can be concluded that the jellium model, complemented by crystallographic orientation-dependent corrections employing the Ashcroft pseudopotential, yields a good physical background of the respective contributions to the work function.

\section{Acknowledgment}

One of the authors (K.F.W.) is indebted to Professor R. Błaszczyszyn for helpful discussions.

\section{References}

[1] N.D. Lang, W. Kohn, Phys. Rev. B 1, 4555 (1970).

[2] N.D. Lang, W. Kohn, Phys. Rev. B 3, 1215 (1971).

[3] H.L. Skriver, N.M. Rosengaard, Phys. Rev. B 46, 7157 (1992) (see also references therein).

[4] R. Monnier, J.P. Perdew, Phys. Rev. B 17, 2595 (1978); 22, 1124 (E) (1980).

[5] R. Monnier, J.P. Perdew, D.C. Langreth, J.W. Wilkins, Phys. Rev. B 18, 656 (1978).

[6] V. Sahni, J.P. Perdew, J. Gruenebaum, Phys. Rev. B 23, 6512 (1981).

[7] Z.Y. Zhang, D.C. Langreth, J.P. Perdew, Phys. Rev. B 41, 5674 (1990).

[8] J.P. Perdew, H.Q. Tran, E.D. Smith, Phys. Rev. B 42, 11627 (1990).

[9] C. Herring, M.H. Nichols, Rev. Mod. Phys. 21, 185 (1949).

[10] T. Agura, Y. Murata, Prog. Surf. Sci. 31, 61 (1989).

[11] J.C. Riviere, in: Work Function: Measurements and Results in Solid State Science, Vol. 1, Ed. M. Green, Decker, New York 1969, p. 6.

[12] J. Hölzl, F.K. Schulte, Work Functions of.Metals, Springer Tracts in Modern Physics, Vol. 85, Eds. G. Hohler, E.A. Niekisch, Springer, Berlin 1979.

[13] A. Kiejna, K.F. Wojciechowski, Prog. Surf. Sci. 11, 293 (1982).

[14] V.S. Fomenko, J.A. Podchernayeva, Emission Properties of Materials, Atomizdat, Moskva 1975 (in Russian).

[15] H.B. Michaelson, J. Appl. Phys. 48, 4729 (1977).

[16] J.E. Alvarellos, E. Chacón, Surf. Sci. 269/270, 590 (1992).

[17] N.W. Ashcroft, D.C. Langreth, Phys. Rev. 155, 682 (1967).

[18] N.W. Ashcroft, D.C. Langreth, Phys. Rev. 159, 500 (1967).

[19] L.N. Dobretsov, M.V. Gomoyunova, Emission Electronics, Nauka, Moskva 1966 (in Russian). 
[20] See Ref. [155] in: N.D. Lang, Solid State Phys. 28, 225 (1973).

[21] R.M. Eastman, C.H.B. Mee, J. Phys. F 3, 1738 (1973).

[22] R. Smoluchowski, Phys. Rev. 60, 661 (1941).

[23] J.K. Grepstad, P.O. Gartland, B. Slagsvold, Surf. Sci. 57, 348 (1976).

[24] A. Mlynczak, R: Niedermayer, Thin Solid Films 28, 37 (1975).

[25] Z. Dworecki, R. Męclewski, J. Cryst. Growth 30, 220 (1975).

[26] R. Błaszczyszyn, M. Błaszczyszyn, R. Męclewski, Surf. Sci. 51, 396 (1975).

[27] Z. Sidorski, I. Pelly, R. Gomer, J. Chem. Phys. 50, 2382 (1969).

[28] A. Kiejna, Phys. Rev. B 47, 7361 (1993).

[29] J.P. Perdew, Phys. Rev. B 21, 869 (1980).

[30] N.W. Ashcroft, Phys. Lett. 23, 48 (1966).

[31] Wei-Mei Shyn, Gaspari, Phys. Rev. 170, 687 (1968).

[32] J.M. Wills, W.A. Harrison, Phys. Rev. B 28, 4363 (1983).

[33] C. Kittel, Introduction to Solid State Physics, 6th ed., Wiley, New York 1986.

[34] S. Anderson, J.B. Pendry, P.M. Echenique, Surf. Sci. 65, 539 (1977).

[35] B.E. Hayden, E. Schweizer, R. Kötz, A.M. Bradshaw, Surf. Sci. 111, 26 (1981). 